\title{
Thermoelastic generation of ultrasound by line-focused laser irradiation
}

\author{
Irene Arias, Jan D. Achenbach * \\ Center for Quality Engineering and Failure Prevention, Northwestern University, Evanston, IL 60208, USA
}

Received 28 October 2002

\begin{abstract}
A two-dimensional theoretical model for the field generated in the thermoelastic regime by line-focused laser illumination of a homogeneous, isotropic, linearly elastic half-space is presented. The model accounts for the effects of thermal diffusion and optical penetration, as well as the finite width and duration of the laser source. The model is obtained by solving the thermoelastic problem in plane strain, rather than by integrating available solutions for the point-source, leading to a lower computational effort. The well-known dipole model follows from appropriate limits. However, it is shown that, by simple elasticity arguments, the strength of the dipole can be related a-priori to the heat input and certain material properties. The strength is found to be smaller than that of the dipoles equivalent to a buried source due to the effect of the free surface. This fact has been overlooked by some previous researchers. Excellent quantitative agreement with experimental observations provides validation for the model. Some representative results are presented to illustrate the generated field and provide insight into the relevance of the different mechanisms taken into account in the model.
\end{abstract}

(c) 2003 Elsevier Ltd. All rights reserved.

Keywords: Laser-generation; Thermoelastic regime; Line-source; Modelling; Non-destructive testing

\section{Introduction}

The irradiation of the surface of a solid by pulsed laser light generates wave motion in the solid material. Since the dominant frequencies of the generated wave motion are generally above $20,000 \mathrm{~Hz}$, the waves are not audible to the human ear, and they are therefore termed ultrasonic waves. There are generally two mechanisms for such wave generation, depending on the energy density deposited by the laser pulse. At high energy density a thin surface layer of the solid material melts, followed by an ablation process whereby particles fly off the surface, thus giving rise to forces which generate the ultrasonic waves. At low energy density the surface material does not melt, but it expands at a high rate and wave motion is generated due to thermoelastic processes. As opposed to generation in the ablation range, laser generation of ultrasound in

\footnotetext{
${ }^{*}$ Corresponding author. Tel.: +1-847-4915527; fax: +1-847-4915227.

E-mail addresses: i-arias@northwestern.edu (I. Arias), achenbach@northwestern.edu (J.D. Achenbach).
} 
the thermoelastic range does not damage the surface of the material. For applications in non-destructive evaluation (NDE), ultrasound generated by laser irradiation in the thermoelastic regime is of interest and will be dealt with in this paper.

The generation of ultrasound by laser irradiation provides a number of advantages over conventional generation by piezoelectric transducers. These are: higher spatial resolution, non-contact generation and detection of ultrasonic waves, use of fiber optics, narrow-band and broad-band generation, absolute measurements, and ability to operate on curved and rough surfaces and at hard-to-access locations. On the receiving side, surface ultrasonic waves can be detected using piezoelectric (PZT) or EMAT transducers, or optical interferometers in a completely laser-based system. Ultrasound generated by laser irradiation contains a large component of surface wave motion, and is therefore particularly useful for the detection of surface-breaking cracks. A scanning laser source technique (SLS) has been proposed by Kromine et al. (2000) for this purpose.

Since White (1963) first demonstrated the generation of high frequency acoustic pulses by laser irradiation of a metal surface, considerable progress has been made in developing theoretical models to explain and provide fruitful interpretation of experimental data. Scruby et al. (1980) assumed that, in the thermoelastic regime, the laser heated region in the metal sample acts as an expanding point volume at the surface, which then was postulated to be equivalent to a set of two mutually orthogonal force dipoles. Based on intuitive arguments, these authors related the strength of the dipoles to the heat input and certain physical properties of the material. In this manner, the thermoelastic circular source was reduced to a purely mechanical point-source acting on the surface of the sample. This point-source representation ne-
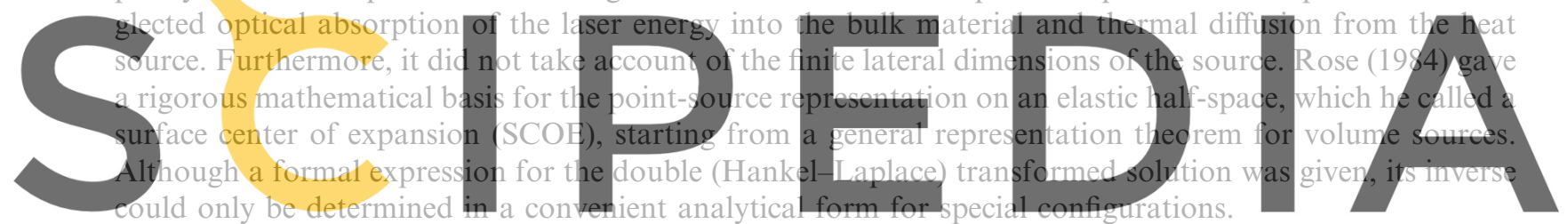

The SCOE model predicts the major features of the waveform and agrees with experiments particularly

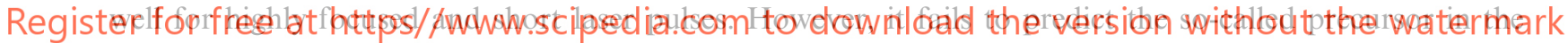
ultrasonic epicentral waveform. The precursor is a small, but relatively sharp initial spike observed in metals at the longitudinal wave arrival. Doyle (1986) proved that the presence of the precursor in metals is due to subsurface sources equivalent to those arising from thermal diffusion. Although the focus was on metallic materials, these results showed that the precursor is present whenever subsurface sources exist. In metals, the subsurface sources arise mainly from thermal diffusion, since the optical absorption depth is very small compared to the thermal diffusion length.

The early work discussed above, suggested that a complete theory based on the treatment of the thermoelastic problem was necessary in order to provide understanding of the characteristics of the generated waveforms and assess the approximations introduced in the formulation of previously proposed models. Based on previous work by McDonald (1989), Spicer (1991) used the generalized theory of thermoelasticity to formulate a realistic model for the circular laser source, which accounted for both thermal diffusion and the finite spatial and temporal shape of the laser pulse.

All the works cited to this point deal with the modelling for a circular spot of laser illumination. One major problem associated with laser ultrasonics is poor signal to noise ratio. By focusing the laser beam into a line rather than a circular spot, the signal to noise ratio can be improved, since more energy can be injected into the surface while keeping the energy density low enough to avoid ablation. In addition, the generated surface waves have almost plane wavefronts parallel to the line-source, except near the ends of the line, which is advantageous for surface crack sizing and for material characterization. Therefore, linesources are used in inspection techniques such as the Scanning Laser Source technique for detection of surface-breaking cracks (Kromine et al., 2000). 
Although the laser line-source offers several advantages, it has received considerably less attention than the circularly symmetric source. Three-dimensional representations for a line of finite width and length can be derived by superposition of surface centers of expansion. In some particular situations, when the effects of thermal diffusion and optical penetration can be neglected and interest is directed only to specific features of the generated field, such as surface wave displacements for instance (Doyle and Scala, 1996), the superposition can be performed readily by analytical integration of the formal expressions put forth by Rose (1984). However, in more general cases, when the finite size of the laser source and the effects of thermal diffusion and optical penetration are accounted for, no analytical solution is available in the physical domain. Then, the superposition has to be performed numerically, resulting in a considerable computational effort. For these more general cases, a two-dimensional approach in which the line-source is considered to be infinitely long becomes highly advantageous. Bernstein and Spicer (2000) formulated a two-dimensional representation for an infinitely long and thin line-source. Their model results in a line of force dipoles acting normal to the line of laser illumination. Thus, they did not consider neither thermal diffusion nor optical penetration nor the finite width of the laser line-source.

In this paper we derive a two-dimensional model for the line-source based on a unified treatment of the corresponding thermoelastic problem in plane strain, rather than integration of available results for the point-source. As opposed to Bernstein and Spicer (2000), this model takes account of the finite width of the source, the shape of the pulse and the subsurface sources arising from thermal diffusion and optical penetration. The thermoelastic problem in an isotropic half-space is solved analytically in the Fourier-Laplace transform domain. The doubly transformed solution is inverted numerically to produce theoretical
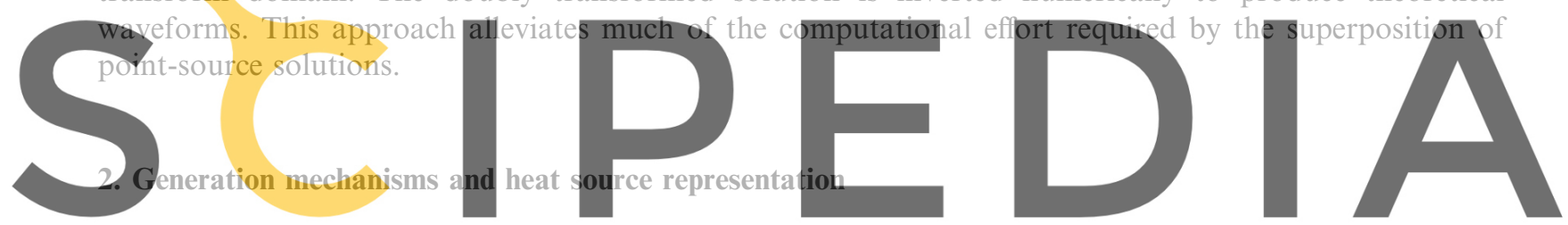

Several physical processes may take place when a solid surface is illuminated by a laser beam depending

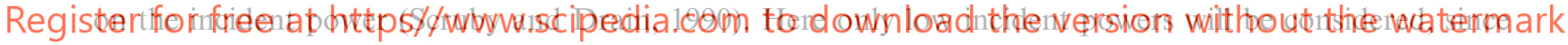
high powers produce damage on the material surface rendering the technique unsuitable for non-destructive testing. At low incident powers the laser source induces heating, the generation of thermal waves by heat conduction, and the generation of elastic waves (ultrasound). In materials such as semiconductors, electric current may be caused to flow.

For application in NDE, generation of elastic waves is required in the ultrasonic frequency range and with reasonable amplitudes. This can be achieved without damage of the material surface only with shortpulsed lasers. The majority of published work has employed $Q$-switched laser pulses of duration of 10-40 ns. A suitable expression for the heat deposition in the solid along an infinitely long line is

$$
q=\hat{E}\left(1-R_{i}\right) \gamma \mathrm{e}^{-\gamma x_{3}} f\left(x_{1}\right) g(t)
$$

with

$$
f\left(x_{1}\right)=\frac{1}{\sqrt{2 \pi}} \frac{2}{R_{\mathrm{G}}} \mathrm{e}^{-2 x_{1}^{2} / R_{\mathrm{G}}^{2}}
$$

and

$$
g(t)=\frac{8 t^{3}}{v^{4}} \mathrm{e}^{-2 t^{2} / v^{2}}
$$

where $\hat{E}$ is the energy of the laser pulse per unit length, $R_{i}$ is the surface reflectivity, $R_{\mathrm{G}}$ is the Gaussian beam radius, $v$ is the laser pulse risetime (full width at half maximum), and $\gamma$ is the extinction coefficient. The 


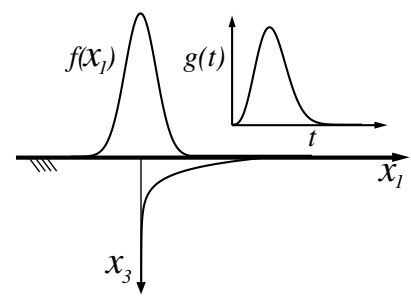

Fig. 1. Spatial and temporal profile of the heat source due to line-focused laser illumination.

temporal and spatial profiles are schematically shown in Fig. 1. The coordinate axis $x_{1}$ and $x_{3}$ are directed along the surface, perpendicular to the line-source and normal inwards from the surface, respectively.

Eq. (1) represents a strip of illumination since it is defined by a Gaussian in $x_{1}$. The Gaussian does not vanish completely with distance, but its value becomes negligible outside a strip. The source is spread out in time according to the function proposed by Schleichert et al. (1989). For both the temporal and the spatial profile, the functional dependence has been constructed so that in the limit $v \rightarrow 0$ and $R_{\mathrm{G}} \rightarrow 0$ an equivalent concentrated line-source is obtained

$$
q=\hat{E}\left(1-R_{i}\right) \delta\left(x_{3}\right) \delta\left(x_{1}\right) \delta(t) .
$$
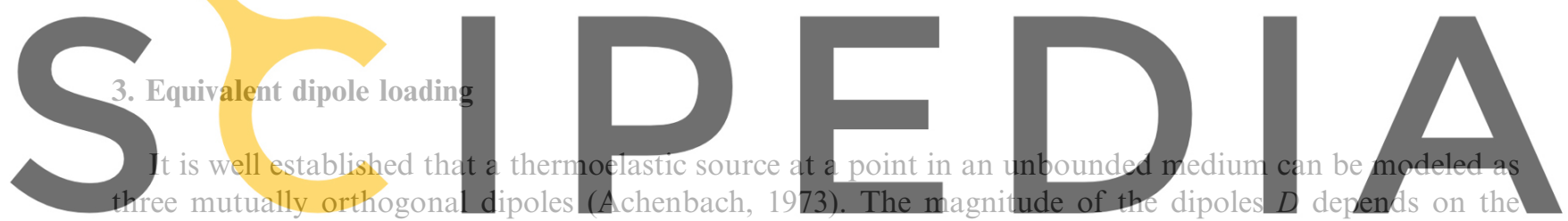

temperature change and certain mechanical and thermal constants of the material. On the basis of intuitive

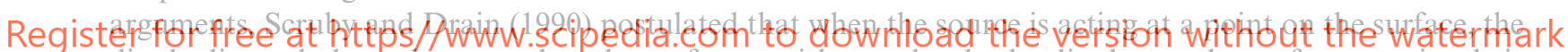
dipole directed along the normal to the surface vanishes and only the dipoles on the surface remain, their strength left unaltered.

We propose a simple approach to derive the magnitude of the surface dipoles. First we simplify the heat deposition process. We consider an instantaneous $(v \rightarrow 0)$ point-source $\left(R_{\mathrm{G}} \rightarrow 0\right.$ in the expression for the corresponding circularly symmetric Gaussian distribution) and assume that all the energy is absorbed at the surface $(\gamma \rightarrow \infty)$. The expression for $q$ then adopts the form, in cylindrical coordinates,

$$
q=E\left(1-R_{i}\right) \frac{\delta(r)}{2 \pi r} \delta\left(x_{3}\right) \delta(t)
$$

where $E$ now represents total energy rather that energy per unit length. This expression can be interpreted as the energy deposited in an infinitesimal circular disc of radius $r_{0}$ and depth $l_{3}$, which both tend to zero. Eq. (5) then becomes

$$
q=E\left(1-R_{i}\right) \frac{1}{\pi r_{0}^{2}} \frac{1}{l_{3}} \delta(t) .
$$

In addition heat conduction or heat propagation is neglected, so that the equation for the temperature reduces to

$$
\frac{\dot{T}}{\kappa}=\frac{E}{k}\left(1-R_{i}\right) \frac{1}{\pi r_{0}^{2}} \frac{1}{l_{3}} \delta(t)
$$


where $T$ represents the absolute temperature, $k$ is the thermal conductivity and $\kappa$ is the thermal diffusivity such that $\kappa=k /\left(\rho c_{V}\right), \rho$ and $c_{V}$ being the density and the specific heat of the material at constant deformation, respectively. Hence the temperature increment in the surface element is

$$
\Delta T=\frac{E}{\rho c_{V}}\left(1-R_{i}\right) \frac{1}{\pi r_{0}^{2}} \frac{1}{l_{3}} H(t) .
$$

Since the dimension of $E / \rho c_{V}$ is temperature (length) ${ }^{3}$, Eq. (8) has the proper dimension for $\Delta T$. Since heat conduction is not considered, the temperature increment $\Delta T$ is maintained at its initial level.

When the laser impinges the surface of the half-space, the very thin circular surface element undergoes thermal expansion due to a temperature increment of $\Delta T$. The element is located at the surface and therefore the normal stress in the $x_{3}$ direction is zero. The elementary disc is shown in Fig. 2(a). If the element is removed from the half-space, it can deform freely in its plane, so that the strains in the radial and circumferential directions are $\alpha_{T} \Delta T$, where $\alpha_{T}$ is the coefficient of linear thermal expansion. To place the element back into the half-space we consider the same surface element subjected to an imposed deformation in its plane of the same magnitude but opposite sign. Let us call this imposed state of strain $\bar{\varepsilon}_{r r}=$ $\bar{\varepsilon}_{\theta \theta}=-\alpha_{\mathrm{T}} \Delta T=\varepsilon$. The corresponding normal stress in the $x_{3}$ direction is

$$
\sigma_{33}=(\lambda+2 \mu) \varepsilon_{33}+2 \lambda \varepsilon=0
$$

\section{and thus}
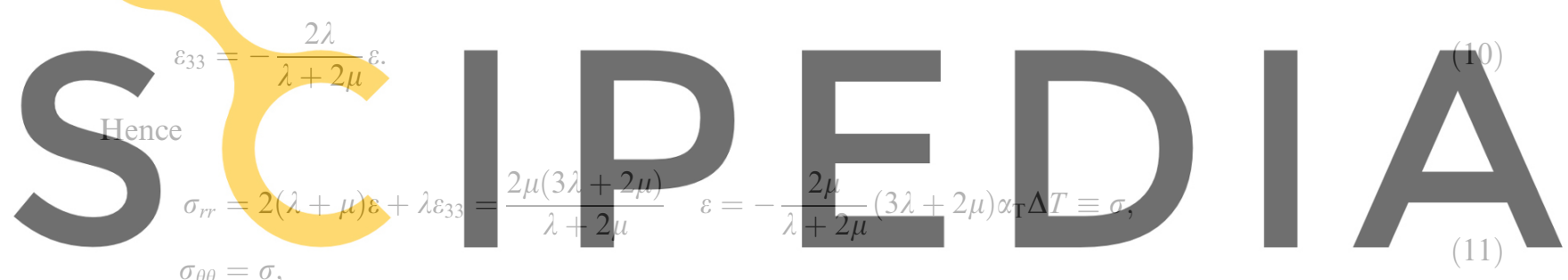

\section{Register for free at https//www.scipedia.com to download the version without the watermark}

where the relevant expression for $\Delta T$ is given by Eq. (8). The reaction of the state of stress given in Eq. (11) on the surface of the hole, shown in Fig. 2(b), is equivalent to that produced by two orthogonai dipoles of magnitude $D$. As illustrated in Fig. 2(a), the force acting on an elementary sector of the circular disc is directed along its normal and its magnitude is

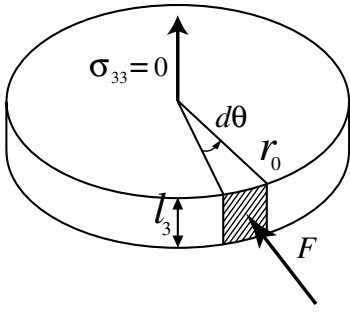

(a)

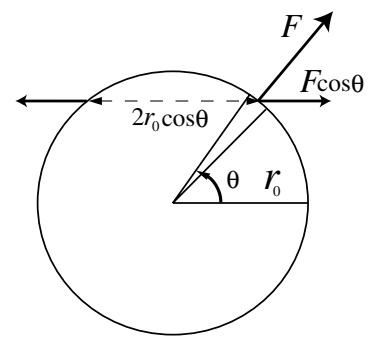

(b)

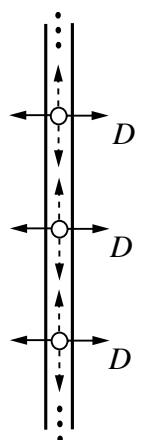

(c)

Fig. 2. Elementary surface disk (a), schematic of forces acting on the surrounding material (b) and schematic of point-source superposition (c). 


$$
F=\sigma \mathrm{d} S=l_{3} \sigma r_{0} \mathrm{~d} \theta .
$$

Consider now two orthogonal directions defined by $\theta=0$ and $\theta=\pi / 2$ in the plane of the element. As shown in Fig. 2(b), the component along one of these directions $(\theta=0)$ of the force acting on the surrounding material is given by $F \cos \theta$. The elementary dipole is obtained multiplying by the separation of the corresponding elementary forces, i.e. $2 r_{0} \cos \theta$, as

$$
\mathrm{d} D=2 r_{0} F \cos ^{2} \theta
$$

and hence, integration along the half-circumference of the hole yields

$$
D=\int_{-\pi / 2}^{\pi / 2} 2 r_{0}^{2} l_{3} \sigma \cos ^{2} \theta \mathrm{d} \theta=\pi r_{0}^{2} l_{3} \sigma .
$$

Thus, finally

$$
D=-\frac{2 \mu}{\lambda+2 \mu}(3 \lambda+2 \mu) \alpha_{\mathrm{T}} \frac{E}{\rho c_{V}}\left(1-R_{i}\right) H(t) .
$$

The same result is obtained for the dipole in the $\theta=\pi / 2$ direction.

The dipole has dimension force-length. Note that Eq. (15) coincides with that given by Scruby and Drain (1990) except for the factor $2 \mu /(\lambda+2 \mu)$. For materials such as aluminum for which $\lambda \simeq 2 \mu$, this factor is approximately 0.5 . The simple derivation presented above shows that, contrary to the conclusion by Scruby and Drain (1990), the free surface does in fact reduce the strength of the surviving dipoles by a factor of $2 \mu(\lambda+2 \mu)$. In a recent work, Royer (2001) reached the same conclusion by comparing a model for an infinitely long and thin line-s obtained by superposition Based on the same argum pendicularly to the axis source based on a mixed matrix formulation to the line-source
point-sources.
of the line. The strength of the dipole can also be derived

elasticity arguments, by obtaining the lateral stresses acting on a very thin surface element which is submitted to $\Delta T$ and is laterally constrained. The resulting expression for the strength of the dipole coincides

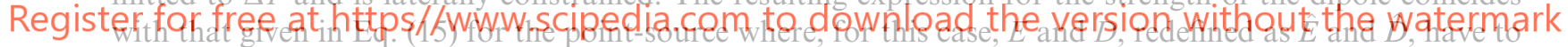

be understood as magnitudes per unit length. This result is not surprising if the line-source is viewed as a superposition of point-sources as shown in Fig. 2(c). The dipoles directed along the axis of the line cancel out, leaving only the dipoles directed normal to the line, their magnitude being unchanged.

\section{Formulation and solution of the thermoelastic problem}

In this section we present the governing equations of the thermoelastic problem with a brief discussion about the appropriate heat conduction representation. We then describe the solution procedure for line illumination of an isotropic half-space. The extension of the formulation to plates is straightforward.

\subsection{Governing equations}

The thermoelastic fields are governed by the coupled equations of thermoelasticity. Based on the hyperbolic generalized theory of thermoelasticity, the governing equations for an isotropic solid are

$$
\begin{aligned}
& k \nabla^{2} T=\rho c_{V} \tau \ddot{T}+\rho c_{V} \dot{T}+T_{0} \beta \nabla \cdot \dot{\mathbf{u}}-q, \\
& \mu \nabla^{2} \mathbf{u}+(\lambda+\mu) \nabla(\nabla \cdot \mathbf{u})=\rho \ddot{\mathbf{u}}+\beta \nabla T,
\end{aligned}
$$


where $T_{0}$ is the ambient temperature, $\mathbf{u}$ is the displacement vector field, $\tau$ is the material relaxation time, $\beta$ is the thermoacoustic coupling constant: $\beta=(3 \lambda+2 \mu) \alpha_{\mathrm{T}}$. In the thermoelastic regime the heat produced by mechanical deformation, given by the term $T_{0} \beta \nabla \cdot \dot{\mathbf{u}}$ can be neglected. With this approximation, Eq. (16) reduces to

$$
\nabla^{2} T-\frac{1}{\kappa} \dot{T}-\frac{1}{c^{2}} \ddot{T}=-\frac{q}{k}, \quad c^{2}=\frac{k}{\rho c_{V} \tau} .
$$

The mathematical expression for $q$ for the case of a line-source is given in Eq. (1). Eq. (18) is hyperbolic because of the presence of the term $\ddot{T} / c^{2}$. On the other hand, its counterpart in the classical theory, i.e. Eq. (18) without the term $\ddot{T} / c^{2}$ is parabolic. In the parabolic description of the heat flow, an infinite heat propagation speed is predicted, while the hyperbolic description introduces a finite propagation speed $c$, which is not known.

Both the classical and hyperbolic heat equations have been used to model thermoelastic laser generated ultrasound. As an accurate determination of the temperature field is vital to accurate predictions of laser generated ultrasonic waves, the question of which equation should be used arises naturally, and has been addressed in the literature by Sanderson et al. (1997).

In order to provide an answer to the question which equation should be used for our particular modelling purposes, the temperature field generated by line illumination of a half-space has been determined based on the two heat equations. Unlike the hyperbolic solution, the classical solution shows no distinct wavefront and temperature increase starts at the initial time as expected. However, the differences in the
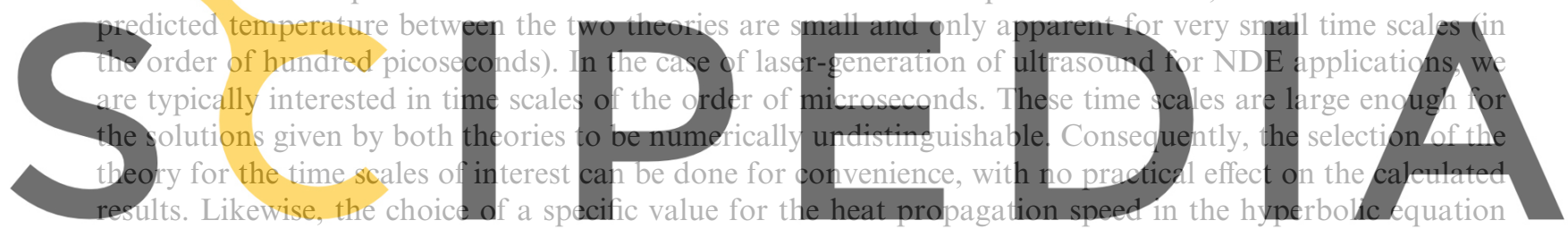

does not affect the results. From the practical point of view, the choice of a value for the heat propagation

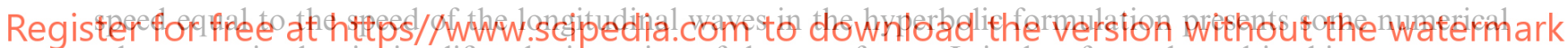
advantages in that it simplifies the inversion of the transforms. It is therefore adopted in this paper.

\subsection{Line-source on an isotropic half-space}

The system of governing equations, which we consider in the plane strain approximation for the case of an infinitely long line-source, must be supplemented by initial and boundary conditions. The initial conditions are that the half-space is initially at rest. The boundary conditions include thermal and mechanical conditions. If the boundary is defined by $x_{3}=0$, then the considered thermal boundary condition is

$$
\frac{\partial T}{\partial x_{3}}=0 \quad \text { at } x_{3}=0
$$

This condition implies that heat does not flow into or out of the half-space via the boundary. The heat that is generated by the laser is deposited inside the half-space just under the surface. The mechanical condition is that the tractions are zero on the surface $\left(x_{3}=0\right)$. The tractions follow from the stress-strain relation

$$
\sigma_{i j}=\lambda \delta_{i j} \epsilon_{k k}+\mu\left(u_{i, j}+u_{j, i}\right)-\beta \delta_{i j} \Delta T .
$$

The term $\beta \delta_{i j} \Delta T$ represents the volumetric stress induced by the temperature change. The conditions of vanishing tractions on the surface become

$$
\sigma_{31}=\mu\left(u_{3,1}+u_{1,3}\right)=0 \quad \text { at } x_{3}=0,
$$




$$
\sigma_{33}=\lambda\left(u_{1,1}+u_{3,3}\right)+2 \mu u_{3,3}-\beta \Delta T=0 \quad \text { at } x_{3}=0 .
$$

Eq. (16) without the mechanically induced heat source, Eqs. (17) and (18) with the above boundary and initial conditions are solved using standard Fourier-Laplace transform techniques for two-dimensional, time dependent systems. First of all, the problem is reformulated in terms of the usual displacement potentials. The elastic displacements may be expressed as

$$
\mathbf{u}=\nabla \phi+\nabla \times \psi
$$

where $\phi$ is the dilatational potential and $\psi=\nabla \times(0,0, \psi)$ is the rotational potential. These potentials satisfy the following wave equations

$$
\begin{aligned}
& \nabla^{2} \phi-a^{2} \ddot{\phi}=\frac{a^{2} \beta}{\rho} T, \\
& \nabla^{2} \psi-b^{2} \ddot{\psi}=0,
\end{aligned}
$$

where $a=1 / c_{\mathrm{L}}$ and $b=1 / c_{\mathrm{T}}$ are the slownesses of the longitudinal and the transverse waves, respectively. The exponential Fourier transform in the spatial coordinate $x_{1}$ and the one-sided Laplace transform in time are then applied to the governing equations, and the boundary and initial conditions. In the transformed domain the thermoelastic problem may then be written as
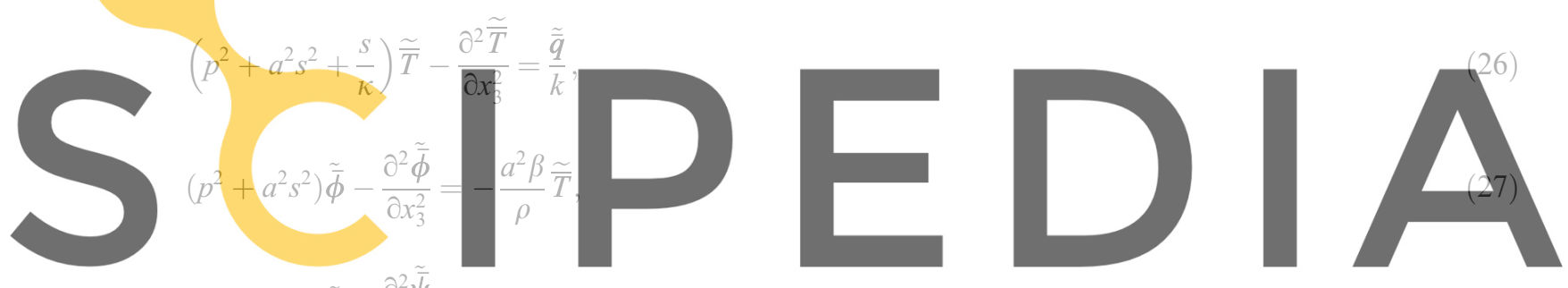

$\left(p^{2}+b^{2} s^{2}\right) \tilde{\bar{\psi}}-\frac{\partial^{2} \bar{\psi}}{\partial s^{2}}=0$

Register for free at https $\xi^{2} /$ www.scipedia.com to download the version without the watermark

with the transformed boundary conditions

$$
\begin{aligned}
& \frac{\partial \widetilde{\bar{T}}}{\partial x_{3}}=0 \quad \text { at } x_{3}=0 \\
& \tilde{\bar{\sigma}}_{33}=\mu\left[\frac{b^{2}}{a^{2}}\left(\frac{\partial^{2}}{\partial z^{2}}-p^{2}\right) \tilde{\bar{\phi}}+2 p^{2}\left(\tilde{\bar{\phi}}+\frac{\partial \tilde{\bar{\psi}}}{\partial z}\right)\right]-\beta \tilde{\bar{T}}=0 \quad \text { at } x_{3}=0 \\
& \tilde{\bar{\sigma}}_{31}=\mu i p\left[2 \frac{\partial \tilde{\bar{\phi}}}{\partial z}+\left(\frac{\partial^{2}}{\partial z^{2}}+p^{2}\right) \tilde{\bar{\psi}}\right]=0 \quad \text { at } x_{3}=0
\end{aligned}
$$

where $(t, s)$ is the Laplace pair and $(x, p)$ is the Fourier pair. The Laplace transformed and the Fourier transformed variables are denoted with an bar and a tilde, respectively. The initial conditions for the transformed potentials are that the body is at rest prior to $t=0$. The thermal problem defined by Eqs. (26) and (29) with the corresponding initial conditions may be solved first for the transformed temperature distribution $\widetilde{\bar{T}}$. This transformed temperature distribution serves as a source term for Eqs. (27) and (28). The transformed potentials can then be obtained by solving the problem defined by Eqs. (27), (28), (30) and (31) with the corresponding initial conditions. The expressions for the transformed displacements and stresses can be derived from the solution for the transformed potentials as 


$$
\begin{aligned}
& \tilde{\bar{u}}_{1}=-i p\left[A \mathrm{e}^{-\zeta x_{3}}-\frac{2 \zeta \eta}{\eta^{2}+p^{2}} A \mathrm{e}^{-\eta x_{3}}+\tilde{\bar{\phi}}_{0}\right], \\
& \tilde{\bar{u}}_{3}=-\zeta A \mathrm{e}^{-\zeta x_{3}}+\frac{2 \zeta p^{2}}{\eta^{2}+p^{2}} A \mathrm{e}^{-\eta x_{3}}+\tilde{\bar{\phi}}_{0}^{\prime}, \\
& \tilde{\bar{\sigma}}_{11}=\mu\left[\zeta \Upsilon(\zeta) A \mathrm{e}^{-\zeta x_{3}}+\frac{4 \zeta \eta p^{2}}{\eta^{2}+p^{2}} A \mathrm{e}^{-\eta x_{3}}+\tilde{\bar{\phi}}_{1}\right], \\
& \tilde{\bar{\sigma}}_{33}=\mu\left[\left(\eta^{2}+p^{2}\right) A \mathrm{e}^{-\zeta x_{3}}-\frac{4 \zeta \eta p^{2}}{\eta^{2}+p^{2}} A \mathrm{e}^{-\eta x_{3}}+\left(\eta^{2}+p^{2}\right) \tilde{\bar{\phi}}_{0}\right], \\
& \tilde{\bar{\sigma}}_{31}=2 \mu i p\left[\zeta A\left(\mathrm{e}^{-\eta x_{3}}-\mathrm{e}^{-\zeta x_{3}}\right)+\tilde{\bar{\phi}}_{0}^{\prime}\right],
\end{aligned}
$$

$$
A=\frac{\left(\eta^{2}+p^{2}\right)^{2}}{R} \Gamma \frac{\gamma^{2}}{\gamma^{2}-\xi^{2}}\left\{\frac{\kappa}{s}\left[\frac{1}{\zeta}-\frac{1}{\xi}\right]+\frac{1}{\zeta^{2}-\gamma^{2}}\left[\frac{1}{\zeta}-\frac{1}{\gamma}\right]\right\},
$$
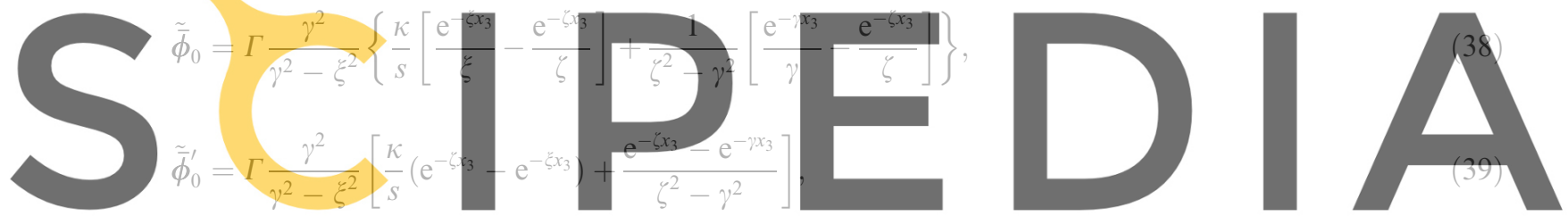

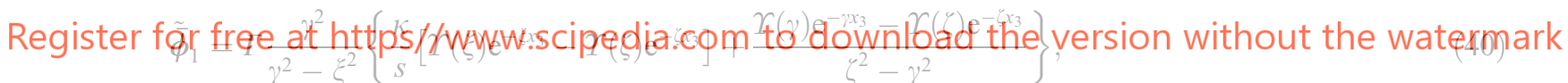

with $\zeta^{2}=p^{2}+a^{2} s^{2}, \eta^{2}=p^{2}+b^{2} s^{2}, \zeta^{2}=\zeta^{2}+s / \kappa$ and $\Upsilon(h)=\left(b^{2} s^{2}-2 h^{2}\right) / h$. Aiso, $R=\left(\eta^{2}+p^{2}\right)^{2}-4 \zeta \eta p^{2}$, $\Gamma=\beta Q_{0} Q_{p}(p) Q_{s}(s) /(\lambda+2 \mu)$ and

$$
\begin{aligned}
& Q_{0}=\frac{\hat{E}}{k}\left(1-R_{i}\right), \\
& Q_{p}(p)=\frac{1}{\sqrt{2 \pi}} \mathrm{e}^{-p^{2} R_{\mathrm{G}}^{2} / 8}, \\
& Q_{s}(s)=\mathscr{L}\left\{\frac{8 t^{3}}{v^{4}} \mathrm{e}^{-2 t^{2} / v^{2}}\right\},
\end{aligned}
$$

where $\mathscr{L}$ indicates Laplace transform. The solution in the transformed domain is then inverted numerically. The integral of the inverse Fourier transform is evaluated by using a Romberg integration routine with polynomial extrapolation (Press et al., 1986). The general method used for the numerical inversion of the Laplace transform is based on a technique developed by Crump (1976).

It is of interest to point out that taking the appropriate limits for an instantaneous $(v \rightarrow 0)$ infinitely thin $\left(R_{\mathrm{G}} \rightarrow 0\right)$ line-source with no optical penetration $(\gamma \rightarrow \infty)$ and no thermal diffusion $\left(\xi^{2} \rightarrow s / \kappa\right)$ the dipole model is recovered. In the above mentioned limits, Eq. (36) takes the form 


$$
\tilde{\bar{\sigma}}_{31}=-\frac{2 \mu}{\lambda+2 \mu}(3 \lambda+2 \mu) \alpha_{\mathrm{T}} \frac{\hat{E}}{\rho c_{V}}\left(1-R_{i}\right) \frac{i p}{s} .
$$

By defining

$$
\hat{D}=-\frac{2 \mu}{\lambda+2 \mu}(3 \lambda+2 \mu) \alpha_{\mathrm{T}} \frac{\hat{E}}{\rho c_{V}}\left(1-R_{i}\right),
$$

Eq. (44) may be rewritten as

$$
\tilde{\bar{\sigma}}_{31}=\hat{D} \frac{i p}{s} \text {. }
$$

The inversion of the Fourier and Laplace transforms yields

$$
\sigma_{31}=\hat{D} \delta^{\prime}\left(x_{1}\right) H(t)
$$

which may be identified as the shear stress induced by a suddenly applied force dipole of magnitude $\hat{D}$ acting on the surface. Taking into account Eq. (45) yields the same expression for the strength of the dipole as given in Eq. (15).

\section{Summary of the relevant models}

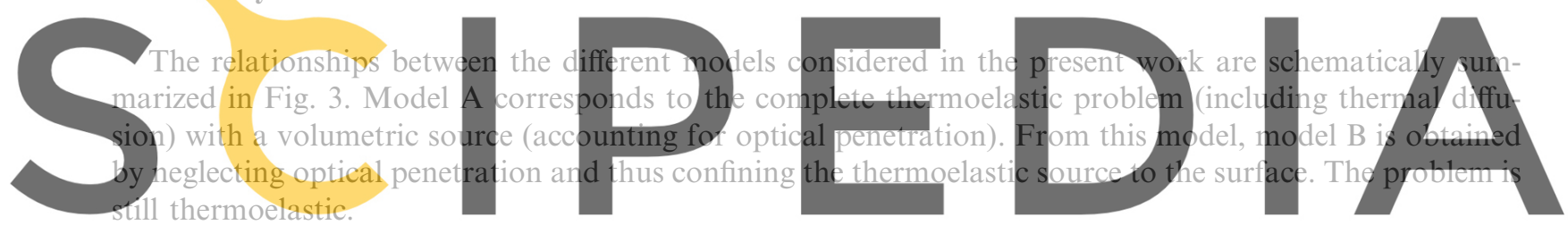

On the other hand, model $\mathrm{C}$ can be obtained from the complete model A by neglecting thermal diffusion.

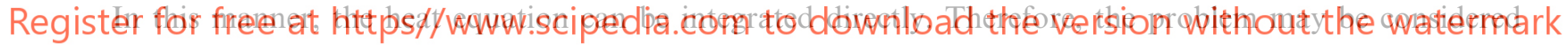

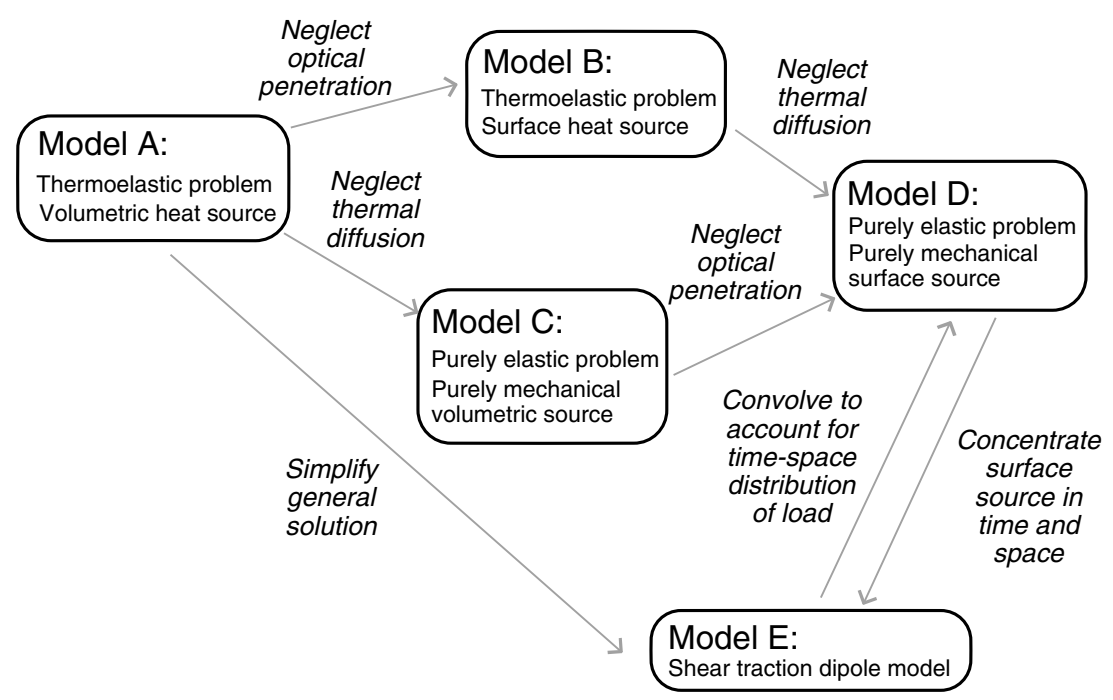

Fig. 3. Schematic summary of the relevant models. 
as equivalent to a purely elastic problem. The source term for the elastic problem obtained by integrating Eq. (7) can be understood as a mechanical volumetric source. Model D can be derived in two different ways, namely by neglecting thermal diffusion in model B or by neglecting optical penetration in model C. In both cases, the resulting model corresponds to the purely elastic problem of a purely mechanical surface load. The shape of the source in the spatial variable $x_{1}$ and in time is the same as the one for the complete model (model A) but is confined to the surface. By concentrating the source to an instantaneous line-source, i.e. by assuming a delta function dependence in both space and time, the shear traction dipole model (model E) is obtained. The procedure of deriving the shear traction dipole model (model E) from the complete model (model A) by neglecting thermal diffusion and optical penetration and concentrating the source in space and time has been demonstrated in detail in the previous section.

It is obvious that model $\mathrm{D}$ may also be obtained from the shear stress dipole model by a convolution with the spatial and temporal distribution of the source. In this sense, the shear traction dipole can be understood as providing a fundamental solution for the purely elastic problem with a surface mechanical load, i.e. when thermal diffusion and optical penetration are neglected.

\section{Representative results}

In this section we present some relevant results obtained with the models described in the previous section. Unless stated otherwise, the values used for the material properties correspond to aluminum alloy 2024-T6 and are: $c_{\mathrm{L}}=6.321 \mathrm{~mm} / \mu \mathrm{s}, c_{\mathrm{T}}=3.11 \mathrm{~mm} / \mu \mathrm{s}, \alpha_{\mathrm{T}}=2.2 \times 10^{-5} \mathrm{~K}^{-1}, \kappa=6.584 \times 10^{-5} \mathrm{~mm}^{2} / \mu \mathrm{s}$, $k=160 \mathrm{~W} / \mathrm{mK}, R_{i}=91 \%, \gamma=2 \times 10^{8} \mathrm{~m}^{-1}$. The values used for the parameters of the laser are: $E=1 \mathrm{~mJ}$ per unit length of the line-source, $R_{\mathrm{G}}=0.45 \mathrm{~mm}$ and $v=10 \mathrm{~ns}$.

\subsection{Quantitative comparison with experiment}

There are not many experimental measurements of ultrasonic waves generated by laser line-sources available in the literature. Most of the data focuses on surface displacements, which are mainly due to the Rayleigh wave. Doyle and Scala (1996) report data of normal surface displacements of Rayleigh waves in an aluminium alloy at several distances along an axis perpendicular to the line-source. They also give precise values of the material properties and the characteristics of the laser source. In this section we present a quantitative comparison between the experimental waveforms reported by Doyle and Scala (1996) and the theoretical waveforms computed with the thermoelastic model (model B). This comparison has been performed without adjusting any parameter in the model to obtain quantitative agreement. Rather, values for the parameters of the thermoelastic model have been taken directly from the experiments. These values are: $c_{\mathrm{L}}=6.42 \mathrm{~mm} / \mu \mathrm{s}, \lambda=57.69 \mathrm{GPa}, \mu=26.45 \mathrm{GPa}, \alpha_{\mathrm{T}}=2.3 \times 10^{-5} \mathrm{~K}^{-1}, \kappa=9.8 \times 10^{-5} \mathrm{~mm}^{2} / \mu \mathrm{s}$, $k=2.38 \times 10^{2} \mathrm{~W} / \mathrm{mK}, R_{i}=91 \%, E=2.9 \mathrm{~mJ}$ per unit length of the line-source, $R_{\mathrm{G}}=10 \mu \mathrm{m}$ and $v=75 \mathrm{~ns}$.

Although the length of the line is obviously finite $(20 \mathrm{~mm})$ in the experiment, the distances of the observation points are close enough for the line-source to be considered infinitely long without significant loss of accuracy. Therefore, as expected for a line-source, the experimental Rayleigh surface wave appears as a monopolar inward displacement which agrees qualitatively with predictions of the thermoelastic model $\left(x_{1}=2.5 \mathrm{~mm}\right.$ in Fig. 5). The quantitative comparison with the experiment has been based on three key features of the Rayleigh pulse shown in Fig. 4, namely the peak amplitude $A$, the full width of the pulse at $1 / e$ of the peak amplitude $\tau_{1}$, and the duration of the pulse $\tau_{0}$. The experimental and the calculated values for these parameters are listed in Table 1. In every case the calculated values are within experimental uncertainty which is evidence of the excellent quantitative agreement between experiment and model. 


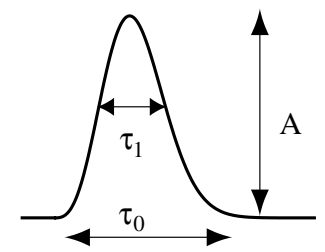

Fig. 4. Key features of the Rayleigh pulse used for the quantitative comparison.
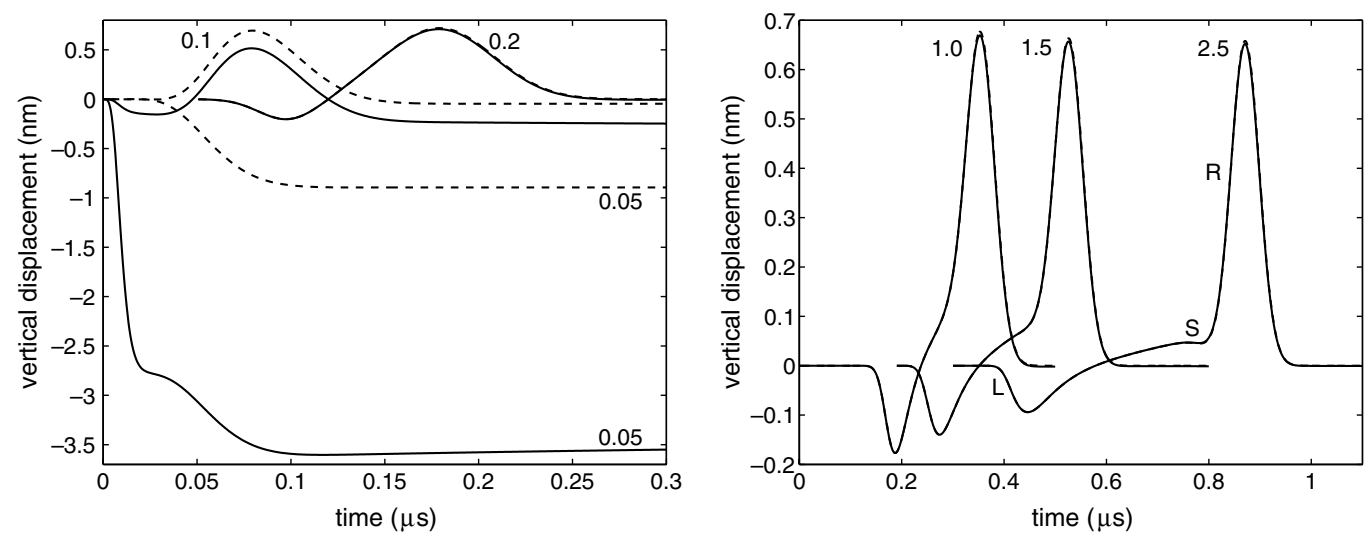

Fig. 5. Vertical displacement on the surface calculated with model B (solid line) and model D (dashed line). The numbers next to the waveforms indicate the distance in $\mathrm{mm}$ from the axis of the laser line-source. The labels $\mathrm{L}, \mathrm{S}$ and $\mathrm{R}$ denote longitudinal, shear and Rayleigh surface waves, respectively.

Table 1

Values of the key features of the Rayleigh waveform

\begin{tabular}{lll}
\hline & Experiment & Theory \\
\hline$A$ & $0.37 \pm 10 \% \mathrm{~nm}$ & $0.35 \mathrm{~nm}$ \\
$\tau_{1}$ & $63 \pm 10 \% \mathrm{~ns}$ & $65 \mathrm{~ns}$ \\
$\tau_{0}$ & $185 \pm 5 \% \mathrm{~ns}$ & $180 \mathrm{~ns}$ \\
\hline
\end{tabular}

\subsection{The effect of thermal diffusion}

In order to identify the effects of thermal diffusion on the predicted waveforms, calculations provided by the model that includes thermal diffusion (model B) are compared with those obtained with the model that only accounts for the spatial and temporal distribution of the laser source (model D). The attention has been directed towards the vertical displacements generated by line-focused laser illumination of an aluminum half-space both on the surface $\left(x_{3}=0\right)$ and on the epicentral axis $\left(x_{1}=0\right)$.

Fig. 5 shows the computed waveforms for vertical displacements on the surface, where a positive displacement is in the positive $x_{3}$ direction, i.e. inwards. The waveforms exhibit a significantly different shape and amplitude depending on the distance from the axis of application of the laser line-source. The portion of the surface which is irradiated undergoes the most extreme variations in displacement. Furthermore, when the point of observation is located well inside the heated region ( $x_{1}=0.05 \mathrm{~mm}$ in Fig. 5), the thermal phenomena taking place right under the source dominate the waveform. Consequently, the disagreement 
between the two predictions is significant, as the purely elastic dipole model (model D) is unable to capture the thermal mechanisms. However, even inside the heated region, the two models show better agreement as the distance to the axis of the line-source increases. The waveforms are undistinguishable for distances larger than $x_{1}=0.6 R_{\mathrm{G}}$.

Experimental measurements of surface vertical displacements generated by a point laser source inside the heated region have been reported in the literature (Spicer and Hurley, 1996) and agree qualitatively with the theoretical waveform for $x_{1}=0.05 \mathrm{~mm}$, the shortest distance shown in Fig. 5. The fact that the experiment is conducted with an axially symmetric source instead on the infinitely long line considered in the calculations does not seem to have a significant effect in the comparison for very short distances relative to the size of the irradiated region.

The above described near-field, i.e. the field generated inside the heated region or very close to it, is of interest in many applications. In particular, one can study theoretically the interactions of the field generated by a scanning laser source with a surface-breaking crack as the source passes over the defect (Kromine et al., 2000). In such a setup, an accurate description of the near-field is vital to quantitative modelling of experimental measurements. The above results show the need for the thermoelastic model.

In the far-field, i.e. well outside the irradiated region $\left(x_{1} \geqslant 1.0 \mathrm{~mm}\right.$ in Fig. 5), the waveform is dominated by the Rayleigh surface wave which travels along the surface without geometrical attenuation. The attenuating longitudinal and shear waves can also be identified in the waveforms for short enough distances. In this region, both models show perfect agreement, as can be expected since the thermal effects are not significant outside a relative small distance from the heated region. The Rayleigh pulse is a monopolar inward displacement, whose temporal profile reproduces that of the laser beam, in contrast with the bipolar Rayleigh pulse produced by a point-source.

Fig. 6 shows the computed waveforms for vertical displacements on the epicentral axis. Again, a positive displacement is in the positive $x_{3}$ direction. The precursor, although small, can be clearly identified in the waveform computed with the thermoelastic model (model B). It is not predicted by the simplified model (model D). For the smaller depths, there is also a disagreement between the two waveform predictions after the arrival of the elastic waves, the signal predicted by the thermoelastic model being weaker. Furthermore, as a consequence of heat diffusion, the temperature field slowly tends to zero with time, and so does the displacement field obtained with model B as shown in Fig. 6 for $x_{3}=0.05 \mathrm{~mm}$. It is clear that, if thermal diffusion is neglected, the heat deposited in the material by the laser will not dissipate and, as follows from Eq. (8), the corresponding temperature field will not vanish as time approaches infinity, even if convolved
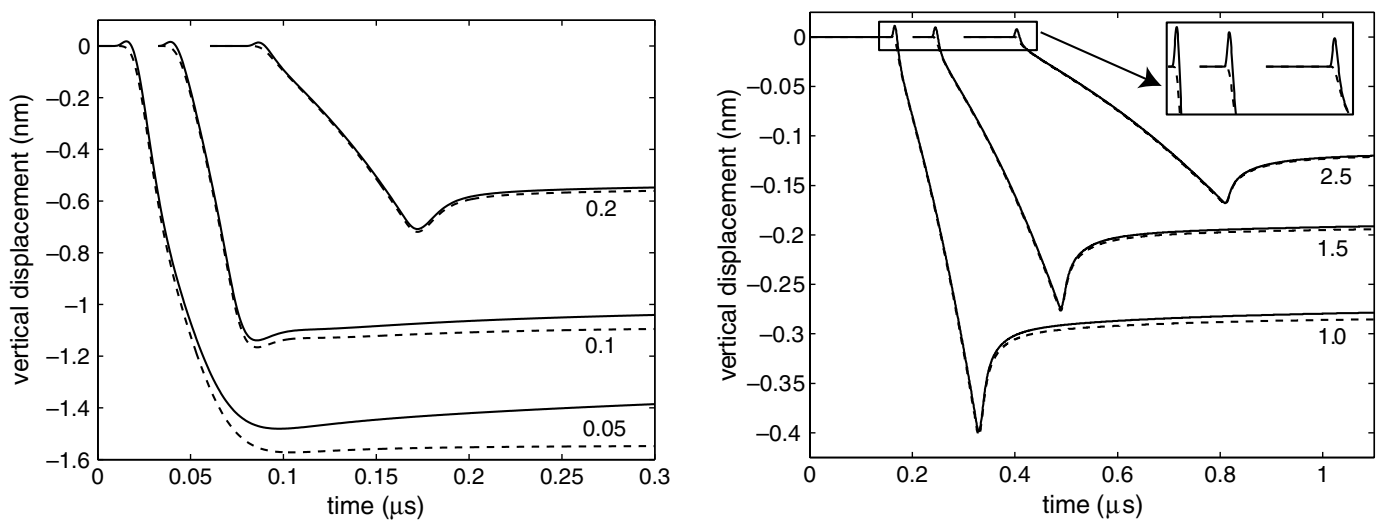

Fig. 6. Vertical displacement on the epicentral axis calculated with model B (solid line) and model D (dashed line). The numbers next to the waveforms indicate the depth in $\mathrm{mm}$. 
with the finite laser pulse temporal profile. Thus, the displacement field obtained with model D exhibits a non-physical, non-zero solution for large times relative to the arrival times of the elastic waves as shown in Fig. 6 for $x_{3}=0.05 \mathrm{~mm}$. Differences between the waveforms predicted by the two models for the time scales of interest are noticeable for distances smaller than around seven times the width of the laser line-source. However, it takes a much larger distance for the disagreement in the precursor part of the waveform to disappear. It should be pointed out that, although the precursor appears to be small relative to the main part of the waveform, it has attracted considerable attention for its potential applications. As a relative sharp, distinct feature of the waveform, it has been used quite effectively for velocity and attenuation measurements. In addition, the precursor may be relevant for calibration purposes. In these applications, a model capable of an accurate prediction of the precursor is of interest.

Although the above results have been obtained for a half-space, one may conclude that in the case of laser-generation in plates, the thickness of the plate relative to the size of the irradiated region will dictate the appropriateness of the use of the simplified model to predict the displacements at the epicenter. For thin plates, the thermoelastic model should be used to accurately determine the epicentral displacement.

\subsection{The effect of the width of the line-source and the duration of the laser pulse}

A parametric study has been carried out to investigate the influence of the width of the line-source $\left(R_{\mathrm{G}}\right)$ and the duration of the pulse $(v)$ on the characteristics of the generated signal. Several waveforms for the vertical displacement at $1 \mathrm{~mm}$ depth on the epicentral axis have been calculated by varying the width of the line-source and the duration of the laser pulse independently and assuming a fixed value for the energy of the laser. The calculated time signals are shown in Fig. 7(a) for the case of increasing width for a fixed pulse duration $(v=10 \mathrm{~ns})$ and Fig. 7(b) for the case of increasing pulse duration for a fixed width $\left(R_{\mathrm{G}}=0.5 \mathrm{~mm}\right)$.

In both cases, as the dimensions of the pulse are increased in space and time, the signal becomes broader and its magnitude decreases. The width of the line-source has also an effect on the amplitude of the final part of the waveform as shown in Fig 7(a). In contrast, this amplitude appears not to be affected significantly by changes in the duration of the laser pulse according to Fig 7(b). In the same figure, a delay in the arrival of the signal can be noted as the energy deposition is spread in time. This effect may be explained by the shift in the position of the peak of the pulse as its duration (v) increases, according to Eq. (3). The same effects are even more noticeable in the shape of the precursor (see insets in Fig. 7). Indeed, for larger or longer laser irradiation, the precursor appears smaller and broader. Its arrival is also delayed for longer
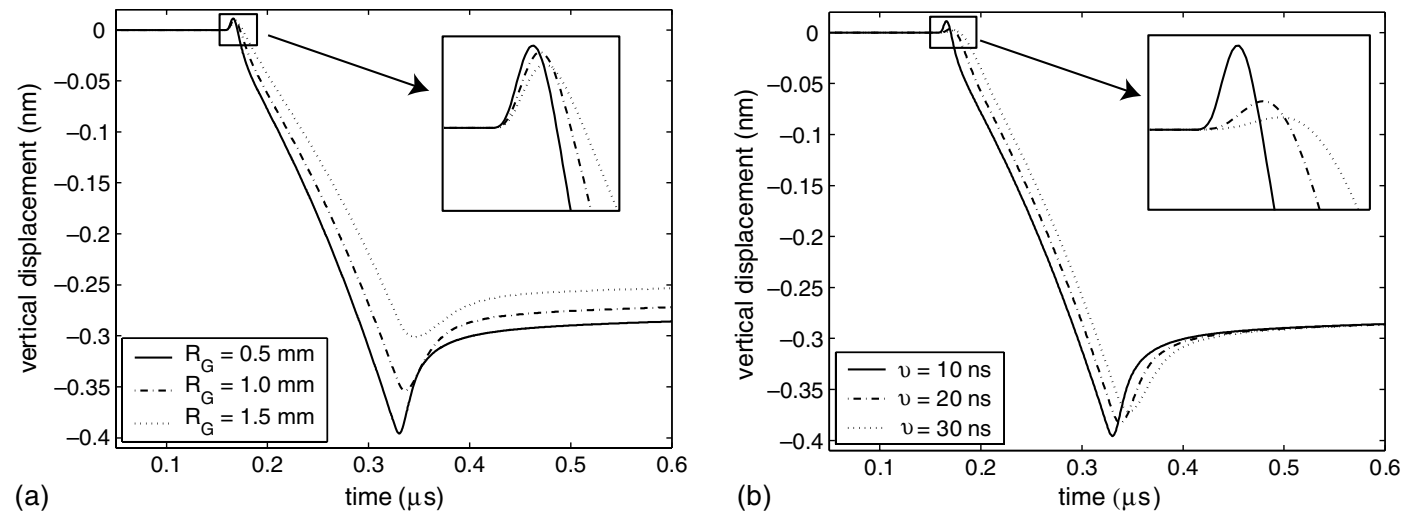

Fig. 7. Influence of the width of the line-source (a) and the duration of the laser pulse (b) on the vertical displacement waveform at the epicentral axis. 
pulse durations. These results agree with those reported in the literature for axially symmetric laser sources (McDonald, 1990).

\subsection{Distribution of stresses}

An understanding of the stress field generated by line-focused laser irradiation may be important for some applications. In particular, one may want to study the interactions of the laser generated field with surface-breaking cracks. For that purpose it is useful to introduce the concept of the scattered field, which is generated by tractions on the faces of the crack such that, when added to those produced by the incident field on the plane of the crack, the condition of traction free crack faces is met. Thus, in order to obtain the scattered field, one needs to know the stresses generated by the incident field, i.e. the field generated by the laser in the absence of the crack. Furthermore, if the laser source is swept across the test specimen as in the scanning laser source technique, the stress field needs to be determined in detail both far and near the source (Arias and Achenbach, 2003).

In this section we describe the stress field generated in an aluminum half-space by line-focused laser illumination. The theoretical results have been obtained with the thermoelastic model which accounts for thermal diffusion (model B), with $R_{\mathrm{G}}=0.45 \mathrm{~mm}$ and $v=10 \mathrm{~ns}$. We present snapshots of the spatial distribution of the stress components $\sigma_{11}, \sigma_{33}$ and $\sigma_{31}$ at different times (Fig. 8) and stress waveforms on the surface (Fig. 9) and on the epicentral axis (Figs. 10 and 11). In these figures, a positive normal stress indicates compression and a positive shear stress on the top face of an element points in the positive $x_{1}$ direction.
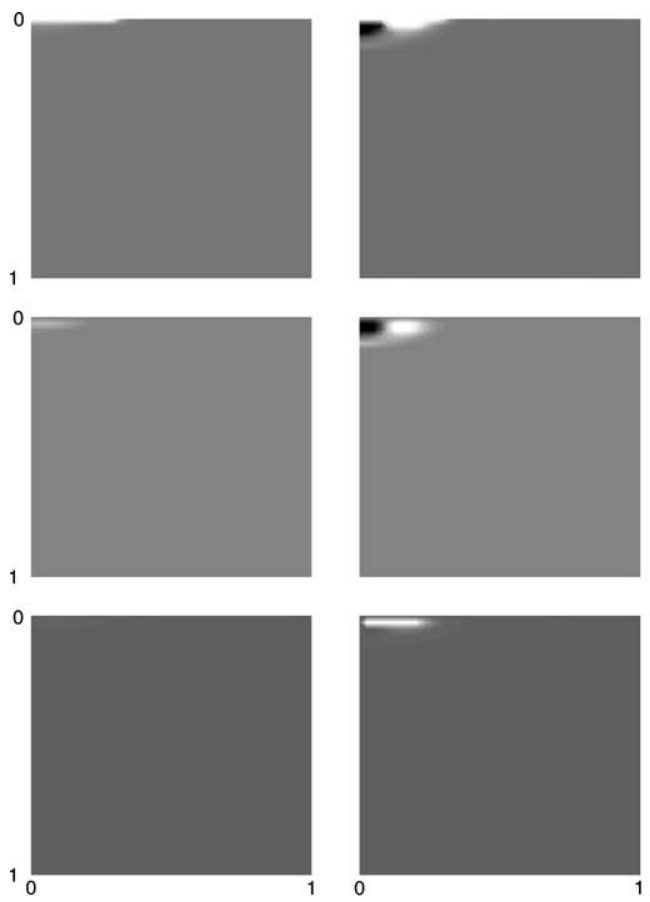
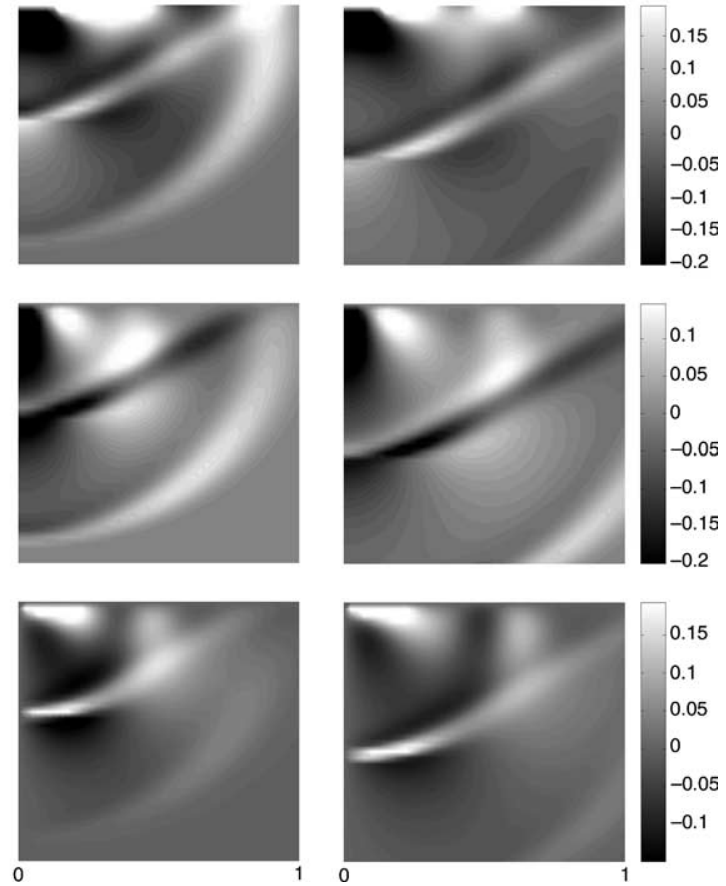

Fig. 8. Snapshots of the stress components $\sigma_{11}$ (top), $\sigma_{33}$ (middle) and $\sigma_{31}$ (bottom) due to the laser line-source at times 0.01 (left), 0.02 (center-left), 0.15 (center-right) and $0.2 \mu \mathrm{s}$ (right), computed for $R_{\mathrm{G}}=0.45 \mathrm{~mm}$ and $v=10 \mathrm{~ns}$. The region shown corresponds to $1 \mathrm{~mm}$ in depth per $1 \mathrm{~mm}$ to the right of the epicentral axis. Positive normal stresses indicate compression. 

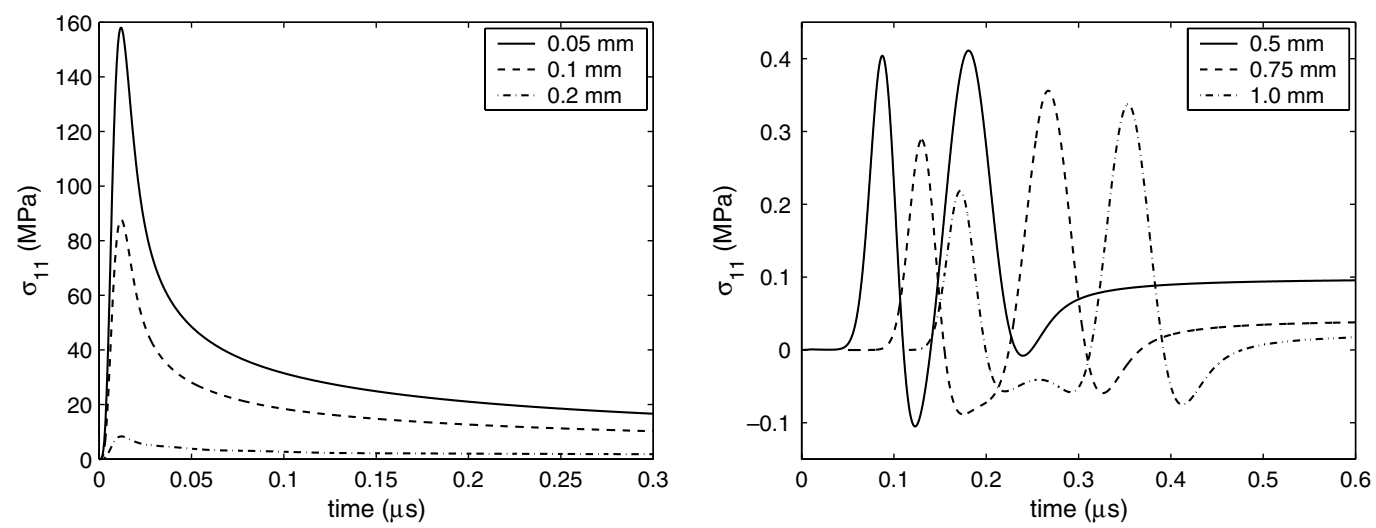

Fig. 9. Stress component $\sigma_{11}$ on the surface. The legend indicates the distance to the epicentral axis. A positive value indicates compression.
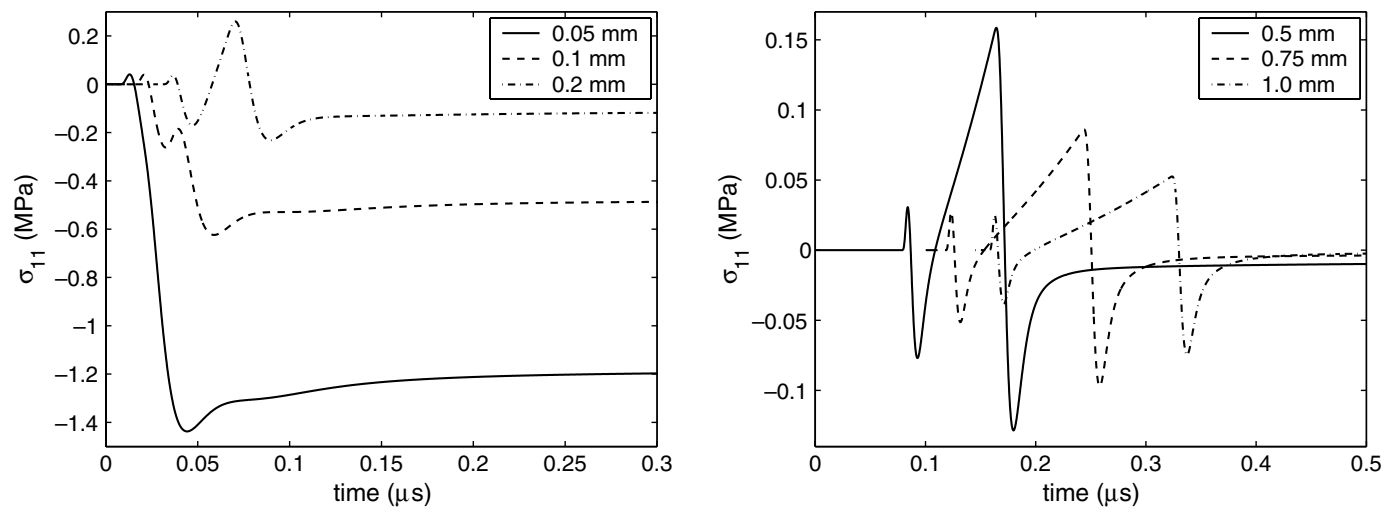

Fig. 10. Stress component $\sigma_{11}$ on the epicentral axis. The legend indicates the depth. A positive value indicates compression.
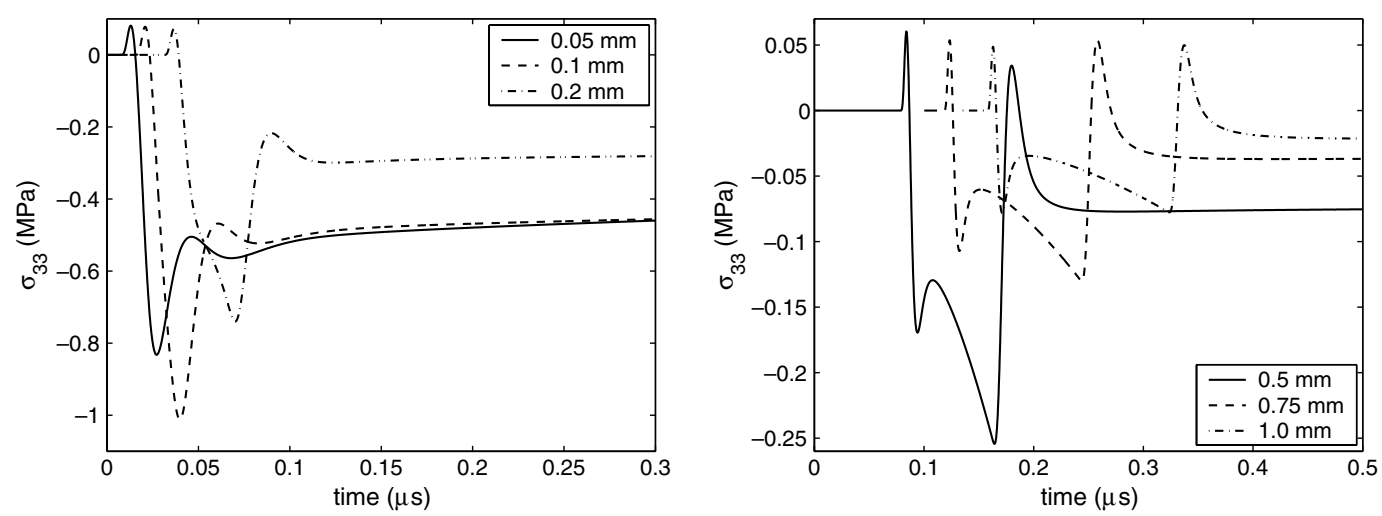

Fig. 11. Stress component $\sigma_{33}$ on the epicentral axis. The legend indicates the depth. A positive value indicates compression. 
The stress field is governed by two phenomena that take place at two very different time scales and exhibit quite different characteristics. Over the duration of the pulse, the laser source deposits heat in a very thin region under the illuminated surface area. The depth of the energy deposition is determined by the thermal diffusion length defined as $l_{\kappa}=\sqrt{4 \kappa v}$. After the pulse, the diffusion of the heat into the bulk of the material takes place at a slow time scale. This phenomenon dominates the stress field in the region near the heat source and gives rise to smooth waveforms with relative high amplitude, such as those shown in Fig. 9 and in Figs. 10 and 11 for $x_{1} \leqslant 0.2 \mathrm{~mm}$ and $x_{3} \leqslant 0.2 \mathrm{~mm}$, respectively. At larger distances from the heat source, the elastic wave propagation resulting from the rapid heat deposition becomes noticeable, as the thermal effects loose intensity. The propagation of elastic waves takes place at a much faster time scale and leads to sharper waveforms with smaller amplitudes as shown in Fig. 9 and Figs. 10 and 11 for $x_{1} \geqslant 0.5 \mathrm{~mm}$ and $x_{3} \geqslant 0.5 \mathrm{~mm}$, respectively.

The snapshots of the spatial stress distribution in the region defined by $1 \mathrm{~mm}$ in depth per $1 \mathrm{~mm}$ to the right of the epicentral axis at four different representative times shown in Fig. 8 provide further illustration for the above described effects. The first snapshots correspond to the instant when the peak of the laser pulse hits the surface $(t=0.01 \mu \mathrm{s})$. At this point, a thin portion of material under the illuminated spot is rapidly heated up. The surrounding material prevents the heated region from expanding laterally which results in a high localized $\sigma_{11}$ compression of the heated material. The highest values of compression appear in the horizontal stress $\sigma_{11}$ at the surface of the heated region. The corresponding waveforms are shown in Fig. 9 for $x_{1} \leqslant 0.2 \mathrm{~mm}$. A smaller $\sigma_{33}$ for compression appears beneath the heated material in the $t=0.01 \mu \mathrm{s}$ snapshot in Fig. 8. This compression can be identified as the precursor in the corresponding near-field $\left(x_{1} \leqslant 0.2 \mathrm{~mm}\right)$ waveforms on the epicentral axis shown in Fig. 11. A small shear stress appears also in this region, although it is not noticeable in the scale of the $\sigma_{31}$ plots in Fig. 8.

A short time after the laser source stops acting on the surface $(t=0.02 \mu$ s in Fig. 8), localized tensions and shear appear beneath the heated region. Intuitively, it is expected that the heated region expands over time both laterally and upwards towards the free surface. On the one hand, the fact that lateral expansion of the heated region is constrained by the surrounding material while the expansion upwards is free, as well as the temperature gradient, induces a slight bending of the thin heated portion of material. This creates a negative pressure under the heated region, thereby giving rise to both vertical and horizontal tensions. These tensions appear as dark spots in the $\sigma_{11}$ and the $\sigma_{33}$ stress maps in Fig. 8. A representative $\sigma_{11}$ waveform for the region under tension is shown in Fig. 10 for $x_{3}=0.05 \mathrm{~mm}$. The waveforms in Fig. 11 for $x_{3} \leqslant 0.2 \mathrm{~mm}$ show this effect for the $\sigma_{33}$ stress component. On the other hand, the lateral expansion of the heated region shears the material under, which is not yet heated, thereby giving rise to the positive shear stress shown in Fig. 8 for $t=0.02 \mu \mathrm{s}$.

At later times $(t=0.15 \mu$ s and $t=0.2 \mu$ s in Fig. 8), the elastic waves have travelled outside the near-field region, where the stress field is dominated by the quasi-static thermoelastic solution. Thus, the elastic wavefronts are noticeable in the plots and in the corresponding far-field waveforms (see Fig. 9 and Figs. 10 and 11 for $x_{1} \geqslant 0.5 \mathrm{~mm}$ and $x_{3} \geqslant 0.5 \mathrm{~mm}$, respectively). In the far-field waveforms for the normal stresses, $\sigma_{11}$ and $\sigma_{33}$, on the epicentral axis (Figs. 10 and 11 for $x_{3} \geqslant 0.5 \mathrm{~mm}$ ), the precursor can be clearly identified as the sharp compressional spike at the arrival of the longitudinal wave. The longitudinal and the head wavefronts appear very clearly, especially in the snapshots of the normal stresses in Fig. 8. As expected, these two wavefronts meet in the surface as can be clearly seen in the $\sigma_{11}$ and $\sigma_{33}$ snapshots for $t=0.15 \mu \mathrm{s}$. While the transverse wavefront is not as distinct in the snapshots of the normal stresses, it is quite visible in the shear stress snapshots. As expected, the head wavefront is tangent to the transverse wavefront. The Rayleigh wavefront can be identified in the $\sigma_{11}$ snapshots in Fig. 8 especially for $t=0.2 \mu$ s where it appears as a light spot on the surface distinct from the quasi-static compression. The far-field $\left(x_{1} \geqslant 0.5 \mathrm{~mm}\right) \sigma_{11}$ waveforms on the surface in Fig. 9 show the Rayleigh wave as a monopolar compression pulse with two smaller tension pulses before and after. At much later times (not shown in Fig. 8), all the heat deposited in the solid eventually dissipates and the quasi-static field vanishes, since the laser pulse is of finite duration. 


\section{Conclusions}

A two-dimensional theoretical model for the field generated in the thermoelastic regime by line-focused laser illumination of a homogeneous, isotropic, linearly elastic half-space has been presented. The model is obtained by solving the corresponding thermoelastic problem in plane strain, rather than by superposition of available three-dimensional solutions for the axially symmetric source, resulting in a smaller computational effort. The thermoelastic problem has been solved by Fourier-Laplace transform techniques. The solutions in the transformed domain have been presented in detail. The inversion of the transforms has been performed numerically to obtain theoretical waveforms.

The model takes account of the effects of thermal diffusion and optical penetration, as well as the spatial and temporal distribution of the source. Each of these effects can be easily neglected in the complete thermoelastic model by taking appropriate limits. By neglecting all of them, the well-known surface dipole model is recovered. Based on simple elasticity considerations, the strength of the dipole has been related to the heat input and certain material properties. The expression differs from that available in the literature by a factor related to the presence of the free surface.

Theoretical waveforms for normal surface displacements due to the Rayleigh wave have been compared with experimental measurements available in the literature and excellent quantitative agreement has been found. This result shows that the proposed thermoelastic model provides a quantitative basis for generation of ultrasound by line-focused laser illumination.

The effect of thermal diffusion has been investigated in vertical displacement waveforms on the epicentral axis and on the surface of an aluminum half-space. As expected, this effect is significant near the heated region, while it is not noticeable in the far-field. If thermal diffusion is neglected in the model, the results have been estimated to be accurate only for distances several times larger than the width of the laser linesource on the epicentral axis and around $60 \%$ of the width of the laser line-source on the surface. The thermoelastic model predicts the precursor spike on the waveforms on the epicentral axis, which results from the subsurface sources arising in metals mainly due to thermal diffusion. A parametric study of the effects of the width of the laser line-source and the duration of the pulse has shown that the generated signal becomes broader and its magnitude decreases as the laser line-source is spread out in space and time. Finally, we have presented stress waveforms on the epicentral axis and at the surface, and snapshots of the stress distribution. These results provide illustration of the different effects under line-focused laser illumination, which have been explained by intuitive arguments.

\section{Acknowledgements}

This paper is based upon work partially supported by the Federal Aviation Administration under Contract \#DFTA 03-98-F-IA029, and partially supported by the Office of Naval Research under Contract N00014-89-J-1362. Irene Arias gratefully acknowledges support from a Fulbright/BSCH Fellowship.

\section{References}

Achenbach, J., 1973. Wave Propagation in Elastic Solids. North-Holland, Elsevier, Amsterdam.

Arias, I., Achenbach, J., 2003. A model for the ultrasonic detection of surface-breaking cracks by the scanning laser source technique, To appear in Wave Motion.

Bernstein, J., Spicer, J., 2000. Line source representation for laser-generated ultrasound in aluminum. Journal of the Acoustical Society of America 107 (3), 1352-1357.

Crump, K., 1976. Numerical inversion of Laplace transforms using a Fourier series approximation. Journal of the ACM 23 (1), 89-96. Doyle, P., 1986. On epicentral waveforms for laser-generated ultrasound. Journal of Physics D 19, 1613-1623. 
Doyle, P., Scala, C., 1996. Near-field ultrasonic Rayleigh waves from a laser line source. Ultrasonics 34, 1-8.

Kromine, A., Fomitchov, P., Krishnaswamy, S., Achenbach, J., 2000. Laser ultrasonic detection of surface breaking discontinuities: scanning laser source technique. Materials Evaluation 58 (2), 173-177.

McDonald, F., 1989. Practical quantitative theory of photoacoustic pulse generation. Applied Physics Letters 54 (16), $1504-1506$.

McDonald, F.A., 1990. On the precursor in laser-generated ultrasound waveforms in metals. Applied Physics Letters 56 (3), $230-232$.

Press, W., Flannery, B., Teulosky, S., Vetterling, W., 1986. Numerical Recipes: The Art of Scientific Computing, third ed. Cambridge University Press.

Rose, L., 1984. Point-source representation for laser-generated ultrasound. Journal of the Acoustical Society of America 75 (3), $723-$ 732.

Royer, D., 2001. Mixed matrix formulation for the analysis of laser-generated acoustic waves by a thermoelastic line source. Ultrasonics 39, 345-354.

Sanderson, T., Ume, C., Jarzynski, J., 1997. Laser generated ultrasound: a thermoelastic analysis of the source. Ultrasonics 35, 115124.

Schleichert, U., Langenberg, K., Arnold, W., Fassbender, S., 1989. A quantitative theory of laser-generated ultrasound. In: Thompson, D., Chimenti, D. (Eds.), Review of Progress in Quantitative Non-destructive Evaluation, vol. 8A. AIP Press, New York, pp. 489496.

Scruby, C., Dewhurst, R., Hutchins, D., Palmer, S., 1980. Quantitative studies of thermally-generated elastic waves in laser irradiated metals. Journal of Applied Physics 51, 6210-6216.

Scruby, C., Drain, L., 1990. Laser Ultrasonics: Techniques and Applications. Adam Hilger, New York.

Spicer, J., 1991. Laser ultrasonics in finite structures: comprehensive modelling with supporting experiment. Ph.D. Thesis, The John Hopkins University.

Spicer, J., Hurley, D., 1996. Epicentral and near epicenter surface displacements on pulsed laser irradiated metallic surfaces. Applied Physics Letters 68 (25), 3561-3563.

White, R., 1963. Generation of elastic waves by transient surface heating. Journal of Applied Physics 34 (12), 3559-3567. 